\title{
Dust in FIR-bright ADF-S galaxies
}

\section{K. Małek ${ }^{1,2}$, A. Pollo ${ }^{2,3}$, T. T. Takeuchi ${ }^{1}$, V. Buat ${ }^{4}$, D. Burgarella ${ }^{4}$ and M. Malkan ${ }^{5}$}

${ }^{1}$ Department of Particle and Astrophysical Science, Nagoya University, Furo-cho, Chikusa-ku, 464-8602 Nagoya, Japan email: malek@cft.edu.pl

${ }^{2}$ National Centre for Nuclear Research, ul. Hoża 69, 00-681 Warszawa, Poland

${ }^{3}$ The Astronomical Observatory, Jagiellonian University, ul. Orla 171, 30-244 Kraków, Poland

${ }^{4}$ Laboratoire d'Astrophysique de Marseille, OAMP, Universite Aix-Marseille, CNRS, 38 rue Frerdeic Joliot-Curie, 13388 Marseille, cedex 13, France

${ }^{5}$ Department of Physics and Astronomy, University of California, Los Angeles, CA 90024, USA

Abstract. Multiwavelength Spectral Energy Distributions (SEDs) of far-infrared (FIR) galaxies detected in the AKARI South Ecliptic Poles Survey (ADF-S) allow to trace differences between [Ultra]-Luminous Infrared Galaxies ([U]LIRGS) and other types of star-forming galaxies (SF).

Keywords. galaxies: infrared - galaxies: evolution - galaxies: spectral energy distribution

\section{Results}

The ADF-S provides the highest quality FIR image of the extragalactic Universe. With its four photometric bands $(65,90,140$, and $160 \mu \mathrm{m})$ it mapps a wide area of $12 \mathrm{sq}^{2}$. We cross-corelated ADF-S catalog with public databases (Małek et al. 2013) and used the CIGALE SED fitting code (Noll et al. 2009) to measure the physical parameters of the ADF-S sources.

We created average SEDs (Fig. 1), normalized all SEDs at rest frame $90 \mu \mathrm{m}$, and divided them into:

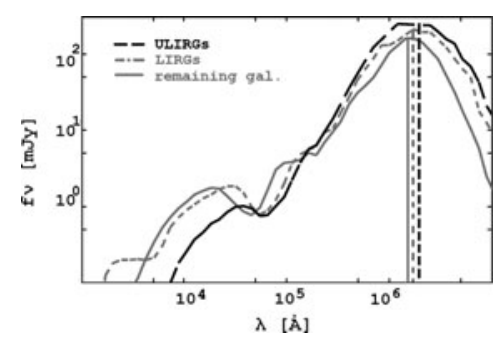

Figure 1. The average SEDs normalized at $90 \mu \mathrm{m}$. ULIRGs (17 galaxies), LIRGs (31 galaxies), and the remaining galaxies ( 82 objects). We notice a significant shift in the peak $\lambda$ of the dust emission in the FIR and a different ratio between luminosities in the optical and IR parts of these three types of galaxies (Małek et al. 2014). It means that [U]LIRGs contain cooler dust that SF galaxies, and that the ratio between luminosities in the optical and IR parts of the spectra increases with the dust luminosity.

\section{Acknowledgements}

AP and KM have been supported by the National Science Centre (UMO-2012/07/B/ST9/ 04425 and UMO-2013/09/D/ST9/04030). KM was supported by the Strategic Young Researcher Overseas Visits Program for Accelerating Brain Circulation No. R2405.

\section{References}

Małek, K., Pollo, A., Takeuchi, T. T., et al. 2013, EPS, Special Issue Cosmic Dust V, 65, 1101

Małek, K., Pollo, A., Takeuchi, T. T., et al. 2014, A\&SA, 562, id.A15

Noll, S., Burgarella, D., Giovannoli, E., et al. 2009, A\&A, 507, 1793 